\title{
A Novel Micro-Nano Structure Profile Control Agent: Graphene Oxide Dispersion
}

\author{
Zhong-Bin Ye, ${ }^{1}$ Yuan Xu, ${ }^{1}$ Hong Chen, ${ }^{2}$ Chen Cheng, ${ }^{1}$ Li-Juan Han, ${ }^{1}$ and Lin Xiao ${ }^{2}$ \\ ${ }^{1}$ Sichuan Province Key Laboratory of Oil and Gas Reservoir of Applied Chemistry, Southwest Petroleum University, \\ Chengdu 610500, China \\ ${ }^{2}$ State Key Laboratory of Oil and Gas Reservoir Geology and Exploitation, Southwest Petroleum University, Chengdu 610500, China
}

Correspondence should be addressed to Yuan Xu; 277619364@qq.com

Received 7 July 2014; Revised 14 October 2014; Accepted 24 October 2014; Published 16 November 2014

Academic Editor: Tianxi Liu

Copyright (C) 2014 Zhong-Bin Ye et al. This is an open access article distributed under the Creative Commons Attribution License, which permits unrestricted use, distribution, and reproduction in any medium, provided the original work is properly cited.

\begin{abstract}
Graphite oxide sheet, now referred to as graphene oxide (GO), is the product of chemical exfoliation of graphite and has been known for more than a century. A GO sheet is characterized by two abruptly different length scales; the apparent thickness of the functionalized carbon sheet is approximately $1 \mathrm{~nm}$, but the lateral dimensions can range from a few nanometers to micrometers. In this paper, an improved method for the preparation of graphene oxide within a mild condition is described. We have found that cancelling the high-temperature stage and prolonging the reaction time of mid-temperature can improve the efficiency of oxidation process. We utilized FTIR, XRD, Ultraviolet-visible, TGA, Raman spectrum, and XPS measurements to characterize the successfully synthesized GO. SEM images were employed to reveal the interior microstructure of as-prepared GO dispersion. We also wondrously found that the GO dispersion could be used as profile control agent in the oilfield water-flooding. Flooding experiments showed that the GO dispersion has an ability to adjust water injection profile, reduce permeability ratio, and improve conformance factor. So the GO dispersion would have potential applications in oilfield exploitation.
\end{abstract}

\section{Introduction}

Recently characterized as "the thinnest material in our universe" [1], graphene, a new class of two-dimensional carbon nanostructure, has stimulated wide interests in both the experimental and theoretical scientific communities due to its extraordinary material properties. In 2004, it was isolated by mechanical exfoliation from graphite crystal and visualized under an optical microscope by Novoselov et al. [2-5]. This has triggered the explosive growth of interest in this new material across many disciplines, which has led to the discovery of many extraordinary properties. For example, graphene was found to have high optical transparency of 97.7\%, high electron mobility of up to $200000 \mathrm{~cm}^{2} \cdot \mathrm{V}^{-1} \cdot \mathrm{s}^{-1}$, high thermal conductivity of up to $5000 \mathrm{~W} \cdot \mathrm{m}^{-1} \cdot \mathrm{k}^{-1}$, high nominal surface area of $2630 \mathrm{~m}^{2} / \mathrm{g}$, and high breaking strength of $42 \mathrm{~N} / \mathrm{m}$. Therefore, many exciting applications have been envisioned and demonstrated [6]. Graphene can be produced by several methods: micromechanical cleavage of nature graphite, chemical vapor deposition (CVD), plasma enhanced CVD, electric arc discharge, epitaxial growth on electrically insulating surface such as $\mathrm{SiC}$, unzipping of carbon nanotubes, and the oxidation-dispersion-reduction [7-9]. Among these methods, the oxidation-dispersionreduction process is widely used for it possesses great potential for large-scale synthesis of graphene. Recently, a number of research groups have devoted themselves to applying graphene in a variety of technological applications including methanol oxidation, lithium-ion battery, solar cell, and transparent conducting film.

Among the family of derivatized graphene sheets, graphite oxide sheets, now called graphene oxide (GO), have actually been made for over a century [10, 11]. Many graphene-based materials can be conveniently synthesized from graphene oxide, which can be prepared in bulk quantities from graphite with strong oxidizing conditions [12-14]. $\mathrm{GO}$ is a layered material featuring a variety of oxygencontaining functionalities with epoxy and hydroxyl groups 


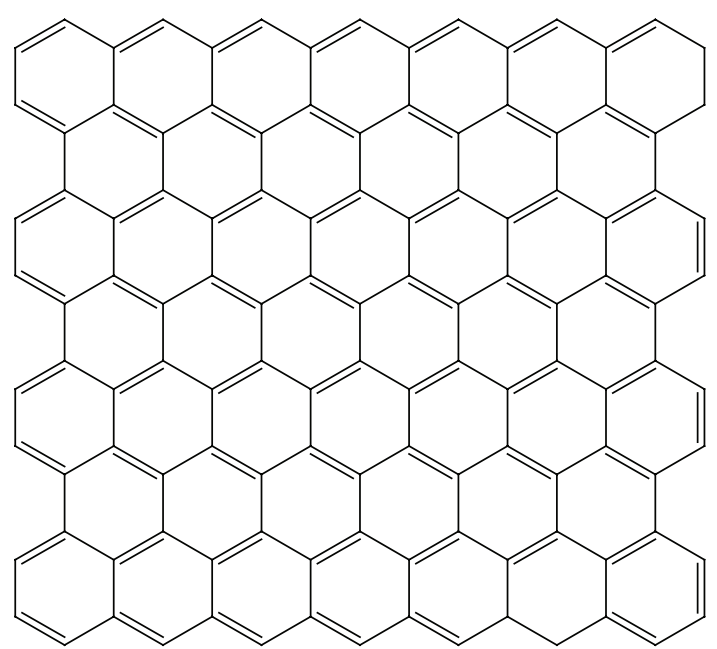

(a)

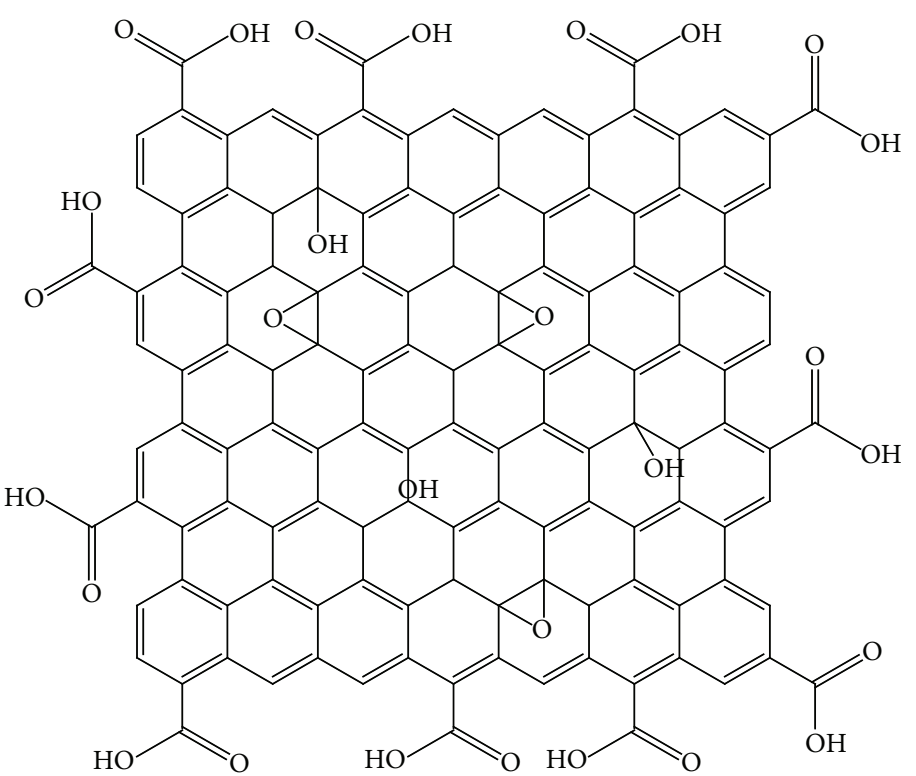

(b)

FIGURE 1: Chemical structural models of graphene-based sheets. (a) Graphene consists of a single atomic layer of sp ${ }^{2}$-hybridized carbon atoms which stack together to form graphite. (b) GO consists of a graphene sheet derivitized by phenyl epoxide and hydroxyl groups on the basal plane and carboxylic acid groups on the edges. The edge-COOH groups can ionize resulting in an electrostatic repulsion between the sheets, allowing single layers to form an aqueous colloidal dispersion [6].

on the basal plane and carbonyl and carboxyl groups along the edges $[15,16]$, which provides a platform for rich chemistry to occur both within the intersheet gallery and along sheet edges [17-19] (Figure 1). These oxygen functionalities render graphene oxide layers hydrophilic and water molecules can readily intercalate into the interlayer galleries. Therefore, GO can be also thought of as a graphite-type intercalation compound with both covalently bound oxygen and non-covalently bound water between the carbon layers. GO is hydrophilic and can readily disperse in water to form colloidal suspensions that are stable for at least months at concentrations of $1 \mathrm{mg} / \mathrm{mL}$ [20].

As shown in the previous reports [21], a GO sheet is characterized by two abruptly different length scales. The thickness is of typical molecular dimensions, measured to be about $1 \mathrm{~nm}$ by atomic force microscopy (AFM). But its lateral dimensions are of common colloidal particles ranging from nanometers up to microns. Therefore, GO sheets can be characterized as either molecules or particles, depending on which length scale/dimension is of greater interest. This molecule-particle duality should naturally make GO a particularly interesting system for both chemists and material scientists.

With the oilfield development, many oil fields have been in medium or high water cut period development stage, comprehensive water cut reached more than $80 \%$, injected water channeling along high permeability layer phenomenon is serious, having the problem of lower water flooding recovery, contradiction of water flooding development is more and more obvious. In order to improve sweep efficiency of oil displacement agent and recovery efficiency, we should adjust profile of fluid absorption. In this paper, we wondrously found that the GO dispersion could be used as profile control agent in the oilfield water-flooding. We successfully synthesized the GO dispersion with the modified Hummer's method and evaluated the performance of the GO dispersion as profile control agent. With regard to the properties of profile control, the GO dispersion would find potential applications in oilfield exploitation.

\section{Materials and Method}

2.1. Materials. Natural graphite powder (purity $\geq 99.85 \%$ ) was supplied by Shanghai Huayi Group Huayuan chemical CO. Ltd., China. Potassium persulfate, phosphorus pentoxide, concentrated sulfuric acid, sodium nitrate, potassium permanganate, hydrogen peroxide, and hydrochloric acid were purchased from Chengdu Kelong Chemical Reagent Factory, China.

\subsection{Preparation of Graphene Oxide (GO) Colloid Dispersion.} Graphene oxide (GO) was synthesized by the modified Hummer's method. The details were described as follows.

In the pretreatment step to ensure oxidation completely, $10 \mathrm{~g}$ of potassium persulfate and $10 \mathrm{~g}$ of phosphorus pentoxide were added in $30 \mathrm{~mL}$ of concentrated sulfuric acid at $80^{\circ} \mathrm{C}$ and stirred slowly until the mixture was dissolved to prepare the oxidation solution. $10 \mathrm{~g}$ of purified natural graphite powder was added to the aforementioned oxidation solution and stirred at $80^{\circ} \mathrm{C}$ for 6 hours. Naturally cooling to room temperature, the solution was diluted with $2.5 \mathrm{~L}$ of deionized 
(DI) water; then, the mixture was filtered and washed with plentiful DI water to remove the residual oxidizing agents and dried in a vacuum oven.

$1 \mathrm{~g}$ of preoxidation graphite powder was dispersed in $23 \mathrm{~mL}$ of concentrated sulfuric acid under stirring at room temperature; then, $0.5 \mathrm{~g}$ of sodium nitrate was added, and the mixture was placed in an ice bath, $3 \mathrm{~g}$ of potassium permanganate was added gradually into the graphite slurry to keep the temperature of the slurry lower than $5^{\circ} \mathrm{C}$ for 2 hours. Successively, the reaction system was heated to $35^{\circ} \mathrm{C}$ in the water bath and kept at the temperature for 2 hours until the slurry became thick, pasty, and brownish. Next, $46 \mathrm{~mL}$ of DI water was added dropwise to the mixture solution, and the temperature was maintained at $35^{\circ} \mathrm{C}$ in the water bath for another 2 hours. Finally, the mixture was diluted with $92 \mathrm{~mL}$ of DI water and treated with 5\% (wt.) hydrogen peroxide solution slowly to terminate the reaction until the mixture changed to brilliant yellow which indicated fully oxidized graphite. Afterward, the mixture was filtered and washed with 5\% (wt.) hydrochloric acid aqueous solution to remove metal ions followed by repeated washing with DI water until a neutral $\mathrm{pH}$ was obtained to remove the excessive acid and inorganic salt. The resulting GO was dried overnight at $60^{\circ} \mathrm{C}$ to produce GO powder. The as-prepared solid graphene oxide was dispersed again in water $(1 \mathrm{mg} / \mathrm{mL})$ by ultrasonication for 2 hours and centrifuged at $8000 \mathrm{rpm}$ for $30 \mathrm{~min}$ to remove aggregates.

2.3. Characterization. The Fourier transform infrared (FTIR) spectra of graphite and GO were recorded using ThermoFisher Scientific Nicolet 6700 over range from 670 to $4000 \mathrm{~cm}^{-1}$. X-ray diffraction (XRD) measurements were performed directly on the powder sample of graphite and GO using PANalytical XPERT-PRO diffractometer $(40 \mathrm{kV}$, $40 \mathrm{~mA})$ with $\mathrm{Cu}(\lambda=1.54 \AA)$ irradiation at a scanning of $2^{\circ} / \mathrm{s}$ in the $2 \theta$ range of $5-70^{\circ}$. The Ultraviolet-visible absorption spectra of GO aqueous dispersion were recorded on a TU1901 double-beam spectrophotometer. Thermogravimetric analysis (TGA) was carried out with NETZSCH STA 449F3. The heating rate was $10^{\circ} \mathrm{C} / \mathrm{min}$. Each time, $\sim 5 \mathrm{mg}$ sample was measured in an aluminum crucible under inert gas atmosphere from 40 to $800^{\circ} \mathrm{C}$. Scanning electron microcopy (SEM) measurements were carried out on a field emission scanning electron microanalyzer (FEI Quanta 450) at an accelerating voltage of $20 \mathrm{kV}$. Raman spectroscopy was performed at room temperature using a HR800 Raman spectrometer at $532.2 \mathrm{~nm}$ wavelength incident laser light. X-ray photoelectron spectroscopy (XPS) analysis was performed with Kratos AXIS Ultra DLD using a monochromic $\mathrm{Al} \mathrm{K} \alpha$ radiation $(h v=1486.6 \mathrm{eV})$ at $15 \mathrm{kV}$ and $10 \mathrm{~mA}$.

2.4. Evaluation of Core Flooding Experiment. Schematic diagrams of experimental set-up were showed in Figure 2. For the flooding experiment of single core, the size of the core was $\varphi 2.5 \times 7 \mathrm{~cm}$, the permeability was $110 \times 10^{-3} \mu \mathrm{m}^{2}$, and the pore volume was $9.9 \mathrm{~cm}^{3}$. The fixed rate of injection was $0.5 \mathrm{~mL} / \mathrm{min}$ and the concentration of GO was $500 \mathrm{mg} / \mathrm{L}$.
We evaluated the flooding property of GO via the pressure of the total injection process.

For the flooding experiment of two cores in parallel, the size of the cores was also $\varphi 2.5 \times 7 \mathrm{~cm}$ and the permeability was $58 \times 10^{-3} \mu \mathrm{m}^{2}$ and $232 \times 10^{-3} \mu \mathrm{m}^{2}$, respectively. The permeability ratio of the cores was $1: 4$. The pore volumes of the cores were $9.5 \mathrm{~cm}^{3}$ and $10.5 \mathrm{~cm}^{3}$, respectively. The fixed rate of injection was $3 \mathrm{~mL} / \mathrm{min}$ and the concentration of GO was $500 \mathrm{mg} / \mathrm{L}$. We evaluated the shunt rate of low permeability layer and the pressure of the total injection process.

\section{Results and Discussion}

GO was synthesized by the traditional Hummer's method including three steps which were low-temperature $\left(0^{\circ} \mathrm{C}\right)$, mid-temperature $\left(35^{\circ} \mathrm{C}\right)$, and high-temperature $\left(98^{\circ} \mathrm{C}\right)$ with concentrated sulfuric acid and potassium permanganate as oxidant. In this paper, we called off the step of hightemperature, which was replaced with prolonging the reaction time of mid-temperature to improve the extent of oxidation of graphite and avoid the damage of eruption in the high-temperature reaction. We could manufacture the GO with a secure and stable condition. The mechanism of the preparation of graphene oxide was described as follows.

In the stage of low-temperature, the mixture presented the color of black because that the oxidizability of concentrated sulfuric acid could not provide the condition of intercalation. Adding potassium permanganate, the edge of graphite begun to be oxidized, which resulted in sulfate ion and strong-polarity vitriol were adsorbed in the edge of graphite with electrostatic attraction. The carbon grid plane of graphite was transformed to positively charged macromolecule plane gradually along with adding of strong oxidation and proceeding of oxidation reaction; thus, negative changed bisulfate ion and strong-polarity vitriol were adsorbed and intercalated into interlayer of graphite to form vitriol-graphite intercalated compound with attractive interaction between charges and electrostatic attraction of strong-polarity vitriol (reaction equation (1)) [22]:

$$
\begin{aligned}
& 6 n \mathrm{H}_{2} \mathrm{SO}_{4}+2 n \text { graphite }+n[\mathrm{O}] \\
& \quad=n \mathrm{H}_{2} \mathrm{O}+2 n\left[\text { graphite }^{+} \cdot \mathrm{HSO}_{4}{ }^{-} \cdot 2 \mathrm{H}_{2} \mathrm{SO}_{4}\right]_{n}
\end{aligned}
$$

In the stage of mid-temperature, the vitriol-graphite intercalated compound was oxidized deeply with strong oxidizability of potassium permanganate, while the oxygen atom and the carbon atom were combined with covalent bond; the partial $\mathrm{C}=\mathrm{C}$ of six-member ring were fractured to form the structure similar with alcohol (reaction equation (2)) [22]:

$$
\begin{gathered}
\mathrm{MnO}_{4}{ }^{-}+2 \mathrm{H}^{+}+\mathrm{SO}_{4}{ }^{2-}+[\text { graphite } \\
\\
\left.\longrightarrow \mathrm{MnO}_{2} \downarrow+\mathrm{HSO}_{4}{ }^{-} \cdot 2 \mathrm{~S}_{2} \mathrm{SO}_{4}\right] \\
+\mathrm{H}_{2} \mathrm{O}+\mathrm{GO}_{y}
\end{gathered}
$$

The mixture was diluted with water after the mid-temperature stage, the vitriol-graphite intercalated compound was hydrolyzed and while vitriol was pushed aside with plenty of water, 


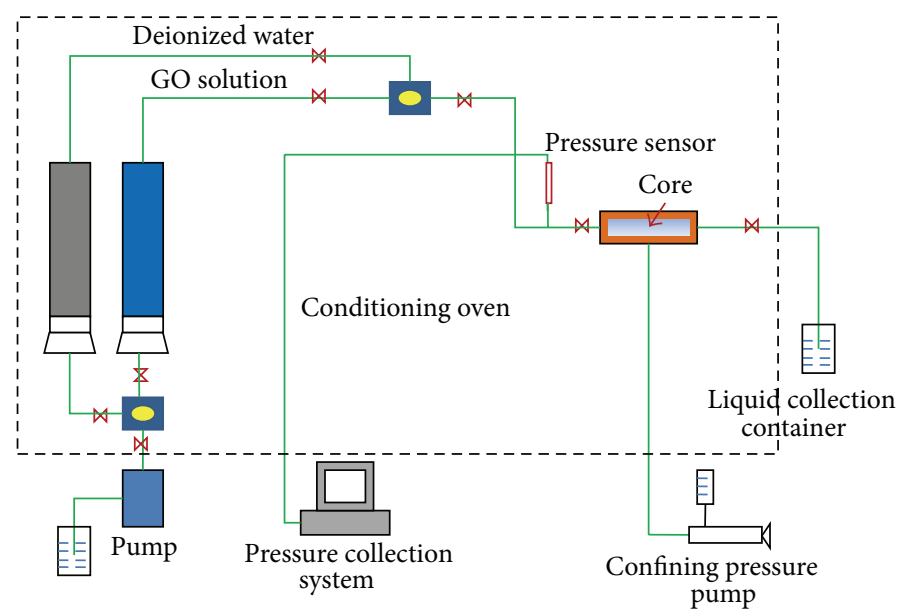

(a)

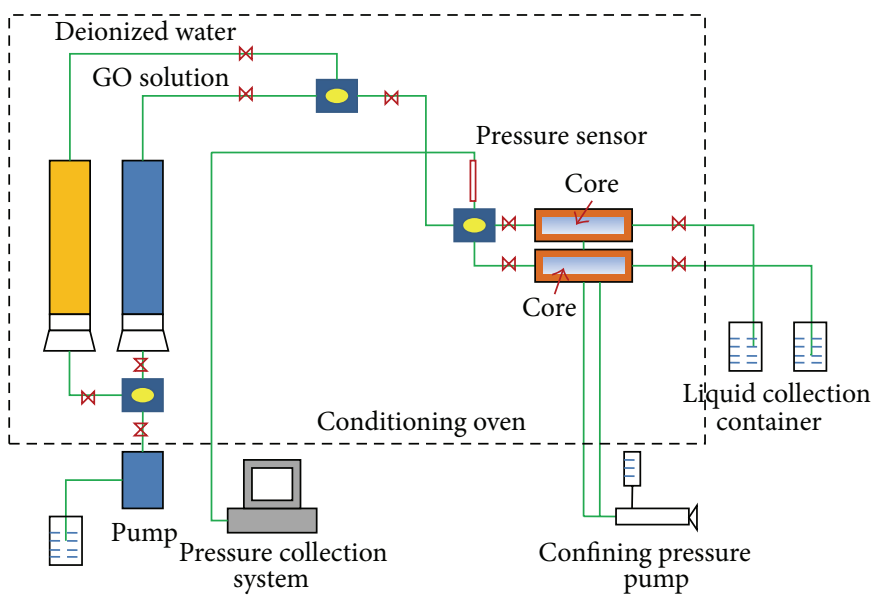

(b)

FIGURE 2: Schematic diagrams of experimental set-up: flooding of single core (a) and flooding of two cores in parallel (b).

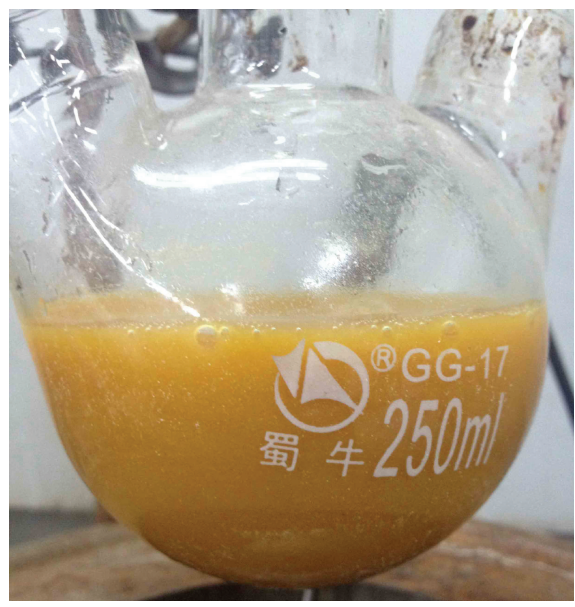

(a)

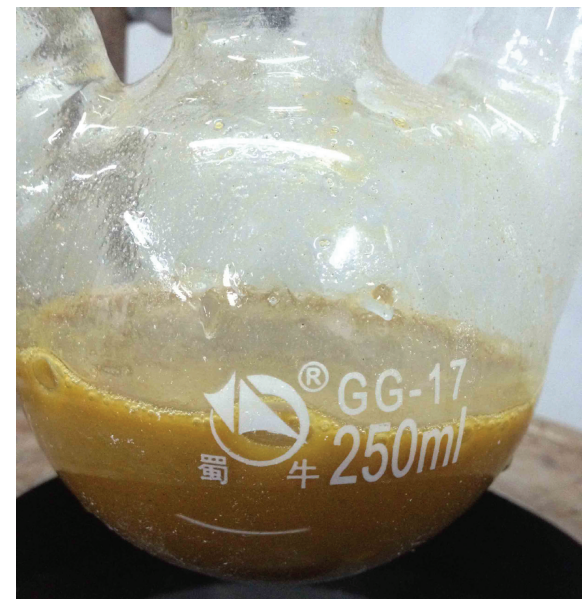

(b)

Figure 3: The GO synthesized by the modified Hummer's method (a) and the traditional Hummer's method (b). 


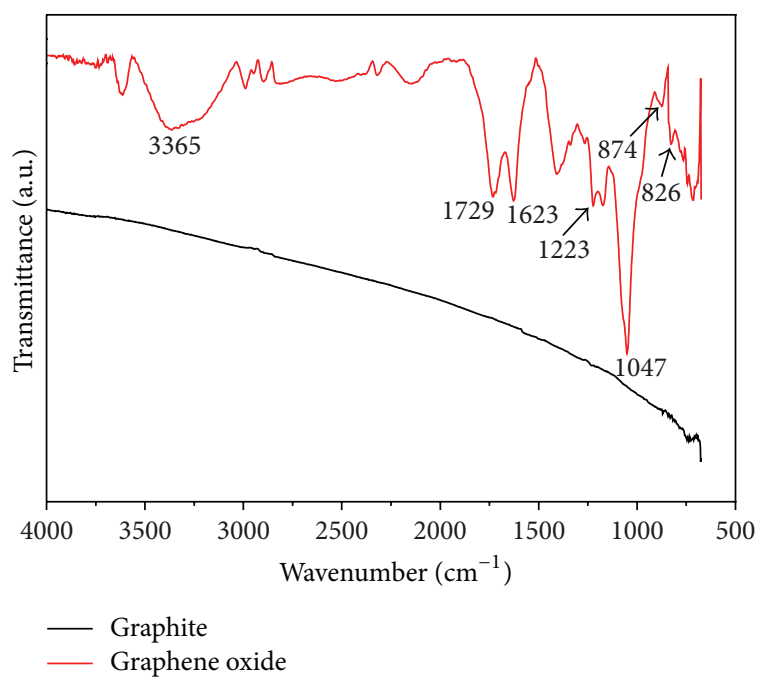

FIGURE 4: FTIR spectra of graphite and GO.

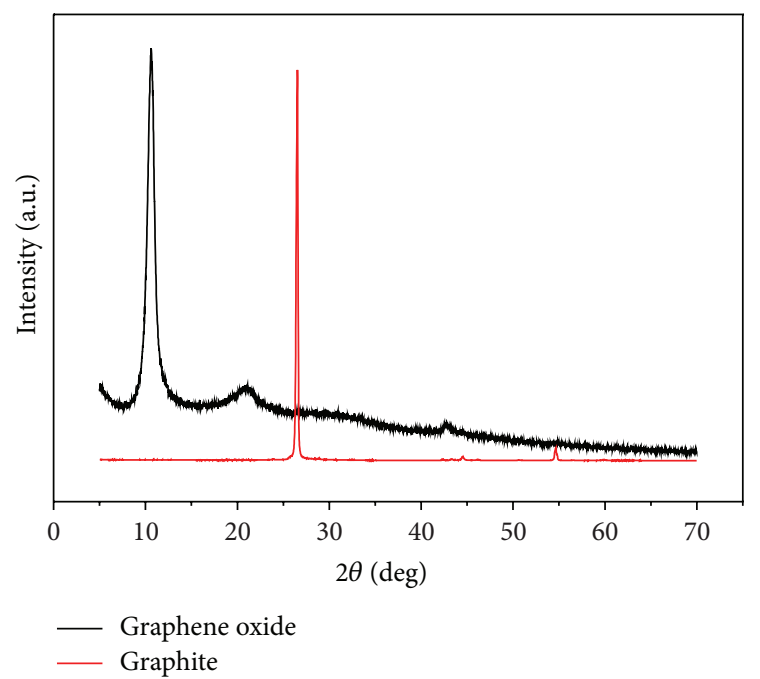

FIGURE 5: XRD patterns of graphite and GO.

hydrogen sulfate ions were exchanged with the hydroxide in the water. In this process, if the temperature was excessive, the vitriol-graphite intercalated compound will be thermally decomposed, and the structure of graphite will be restored rapidly, resulting in that GO could not be formed on account of that the hydroxide and water could not enter into interlamination of graphite. Thus, during the above-mentioned stage, the thermal decomposition reaction would reduce the oxidation degree of graphite because of the excessive temperature. The GO synthesized by the traditional Hummer's method (T-GO) and the modified Hummer's method (M-GO) were shown in Figure 3. M-GO was oxidized more adequately than T-GO with a brighter color. So in our study, we canceled the high-temperature reaction and maintained $35^{\circ} \mathrm{C}$ to carry out the last step reaction after adding the water dropwise to the mixture solution.

FTIR spectra of pristine graphite and GO were presented in Figure 4. For the graphite spectrum, no vibration peak was

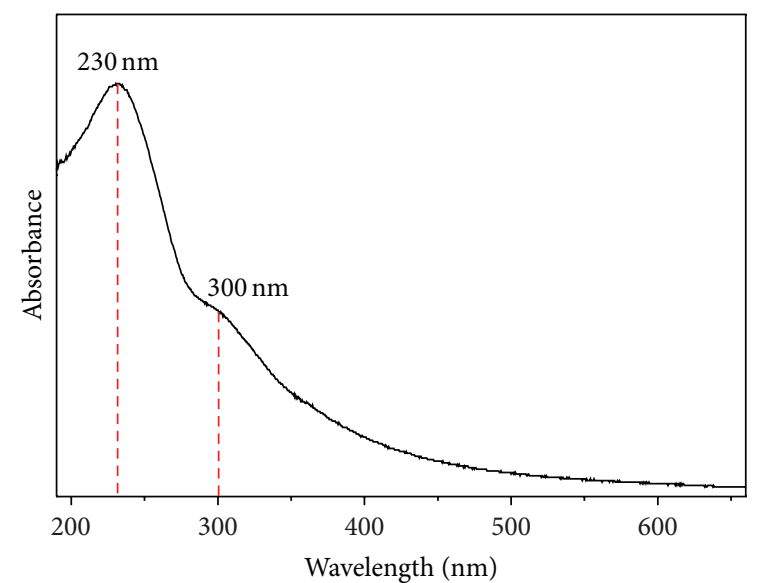

FIGURE 6: UV-Vis absorption spectra recorded in aqueous dispersion at $0.05 \mathrm{mg} / \mathrm{mL}$ of $\mathrm{GO}$.

observed. The FTIR spectra of GO was tested for confirming the successful synthesis of GO. The intense band at $3365 \mathrm{~cm}^{-1}$ and the broad band at $3200 \mathrm{~cm}^{-1}$ to $3700 \mathrm{~cm}^{-1}$ were attributed to stretching of the $\mathrm{O}-\mathrm{H}$ bond of $\mathrm{CO}-\mathrm{H}$ and residual water molecules, which confirmed that the GO could not be dried thoroughly. The band at $1729 \mathrm{~cm}^{-1}$ was associated with stretching of the $\mathrm{C}=\mathrm{O}$ bond of carbonyl or carboxyl groups. The bands present at 1623 and $1223 \mathrm{~cm}^{-1}$ were attributed to deformations of the $\mathrm{O}-\mathrm{H}$ bond in water and $\mathrm{CO}-\mathrm{H}$ groups, respectively. Deformation of the $\mathrm{C}-\mathrm{O}$ bond was observed as the intense band present at $1047 \mathrm{~cm}^{-1}$. The bands at 874 and $826 \mathrm{~cm}^{-1}$ were attributed to characteristic absorption band of the $\mathrm{C}-\mathrm{O}-\mathrm{C}$.

XRD provided a conclusive proof for the degree of oxidation as the layer distances of pristine graphite and GO was highly different which was showed in Figure 5. For the pristine graphite, the (002) diffraction peak appears at $2 \theta=26.5^{\circ}$, indicating an interlayer spacing of $0.34 \mathrm{~nm}$ between graphene platelets, obtained by Bragg's equation. After oxidization-induced expansion, the (002) diffraction peak shifted to $2 \theta=10.6^{\circ}$, with no reflections at $26.5^{\circ}$, suggesting that the interlayer distance increased to $0.834 \mathrm{~nm}$ due to oxidation and completely eliminated the $0.34 \mathrm{~nm}$ graphite interlayer spacing. This suggested that the graphite was efficiently oxidized. Increase in the interlayer spacing was attributed to the introduction of oxygenic functional groups and the presence of water molecules between the graphite layers or other structural defects [23].

The Ultraviolet-visible absorption spectra of GO aqueous dispersion were showed in Figure 6. The absorption peak at $230 \mathrm{~nm}$ of GO corresponded to $\pi \rightarrow \pi^{*}$ transitions of aromatic $\mathrm{C}=\mathrm{C}$ bonds and the absorption peak at $300 \mathrm{~nm}$ corresponded to transitions of $\mathrm{C}=\mathrm{O}$ bonds.

The thermal stability of the GO in comparison with pristine graphite, characterized by TGA, was showed in Figure 7. The TGA curve of GO displayed two prominent mass losses at about $100^{\circ} \mathrm{C}$ and $200^{\circ} \mathrm{C}$. The TGA curve of GO showed about $11 \mathrm{wt} \%$ mass loss at $100^{\circ} \mathrm{C}$, which could be attributed to the evaporation of water trapped between 


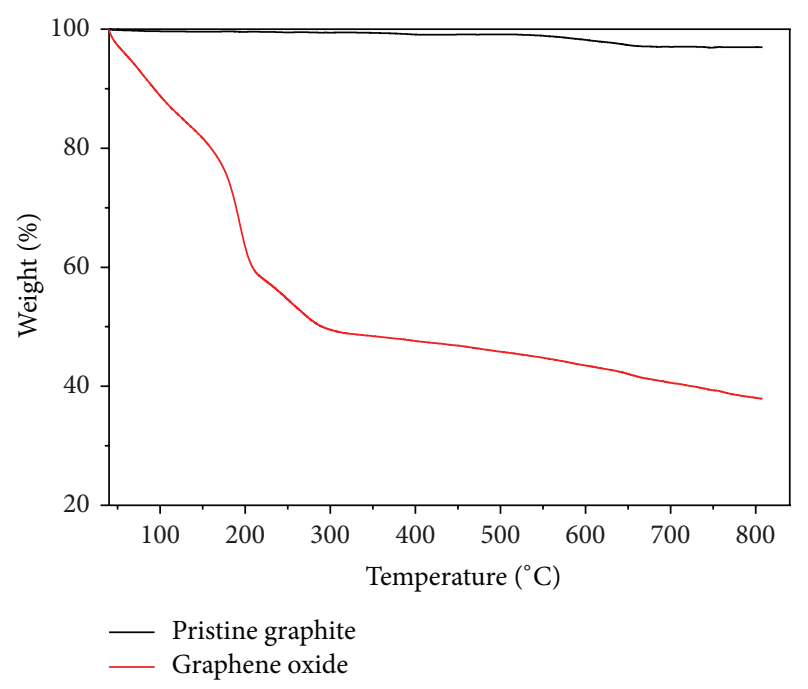

Figure 7: TGA curves of graphite and GO.

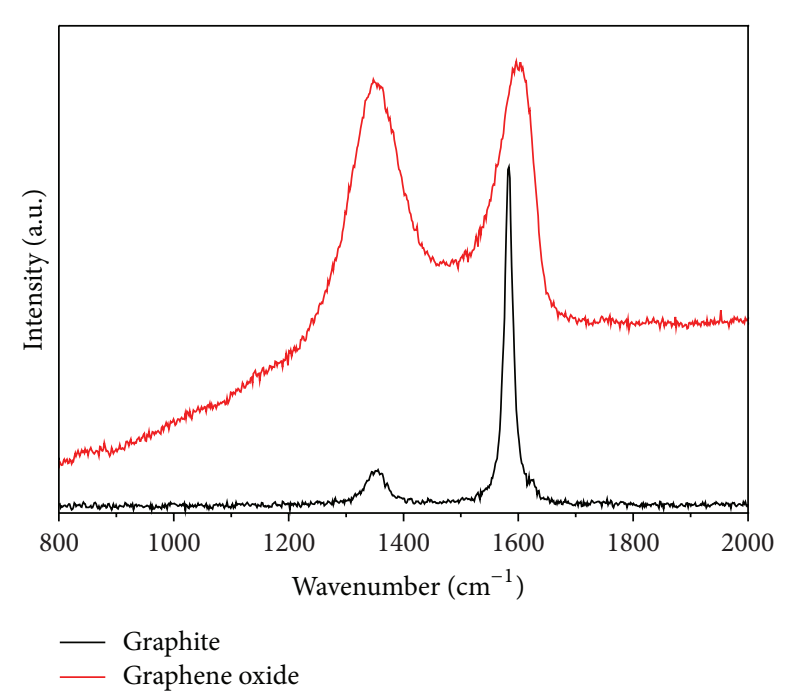

FIGURE 8: Raman spectra of graphite and GO excited at $532.2 \mathrm{~nm}$.

hydrophilic GO sheet. It further demonstrated that GO could not be dried thoroughly in accordance with the result of FTIR. And the main mass loss occurred from $150^{\circ} \mathrm{C}$ to $250^{\circ} \mathrm{C}$ due to the thermal decomposition of oxygen-containing functional groups from the graphene oxide surface such as hydroxyl, epoxy, and carbonyl groups which verified that the graphite had been oxidized successfully. The GO loss was approximately $62 \mathrm{wt} \%$ at $800^{\circ} \mathrm{C}$. However, the TGA curve of the pristine graphite indicated approximately $3 \mathrm{wt} \%$ mass loss at $800^{\circ} \mathrm{C}$ and nearly no mass loss under $100^{\circ} \mathrm{C}$ which testified that the graphite was super-hydrophobic and had a thermodynamic stability.

Raman spectroscopy was a powerful approach to characterize the graphite degree of carbon-based materials. The significant structural changes occurred during the chemical processing from pristine graphite to GO, was also reflected in their Raman spectra. Figure 8 showed the Raman spectra

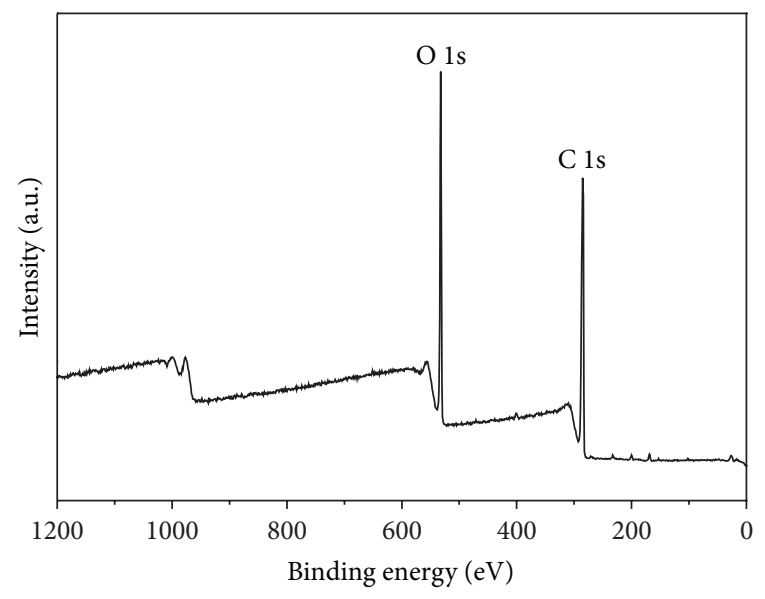

(a)

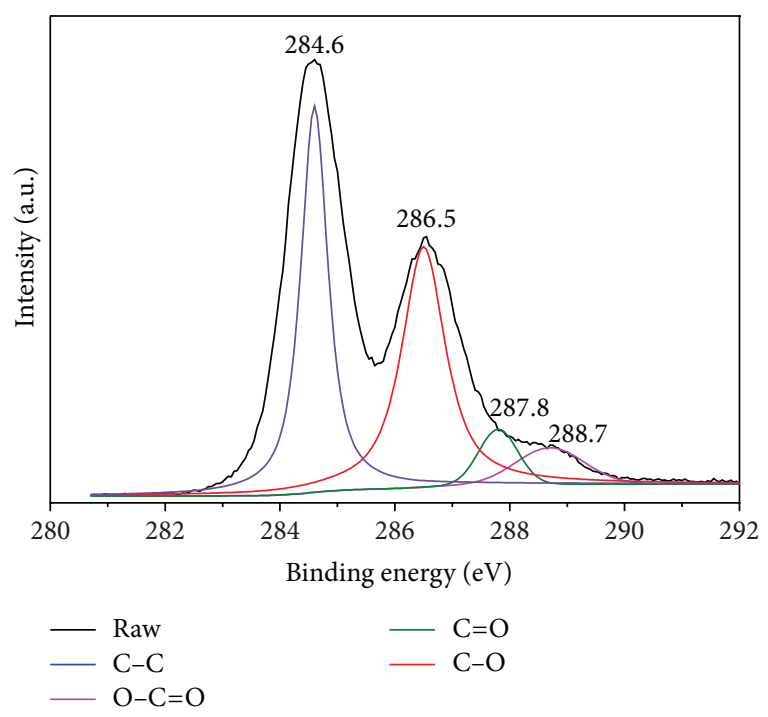

(b)

FIGURE 9: XPS spectra of GO (a) and high resolution scans of $\mathrm{C}$ 1s region of $\mathrm{GO}(\mathrm{b})$.

of natural graphite and GO. It was well recognized that the $\mathrm{D}$ band resulted from a breathing mode of $\kappa$-point photons of $A_{1 g}$ symmetry, while the $G$ band was due to the first order scattering of the $\mathrm{E}_{2 \mathrm{~g}}$ phonon of $\mathrm{sp}^{2} \mathrm{C}$ atoms [24]. The $\mathrm{D}$ band of natural graphite was located at $1355 \mathrm{~cm}^{-1}$. The $\mathrm{G}$ band of natural graphite was located at $1583 \mathrm{~cm}^{-1}$ and resulted from the in-phase vibration of the graphite lattice. After oxidizing, the intensity of the $\mathrm{D}$ band was increased, and the $\mathrm{D}$ band of $\mathrm{GO}$ was located at $1347 \mathrm{~cm}^{-1}$, which resulted from the decrease in the size of the in-phase $\mathrm{sp}^{2}$ domains due to the extensive oxidation. The $\mathrm{G}$ band of GO became much broader and shifted to $1596 \mathrm{~cm}^{-1}$ owing to the present of isolated double bonds that resonated at the higher frequencies than the $\mathrm{G}$ band of graphite [25].

XPS measurement was applied to investigate the chemical bonds formed on the surface of GO. The survey XPS data was showed in Figure 9(a). There were two obvious peaks 


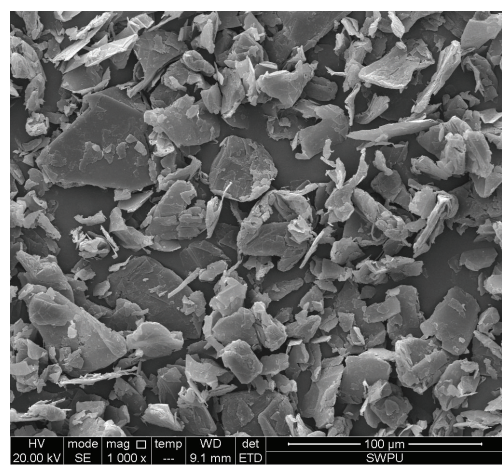

(a)

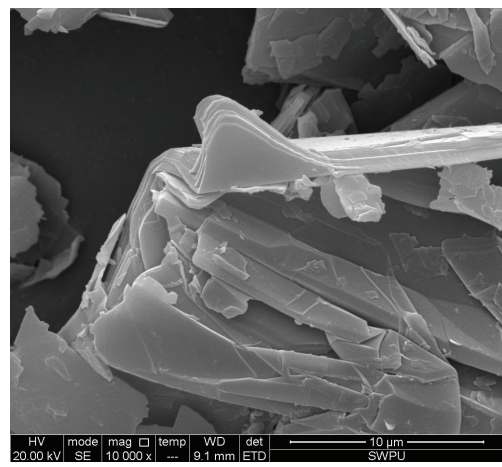

(d)

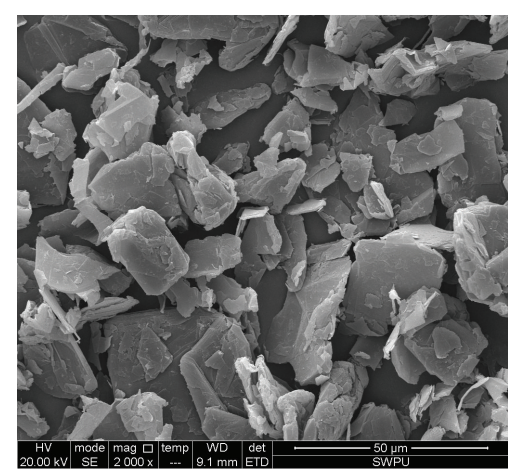

(b)

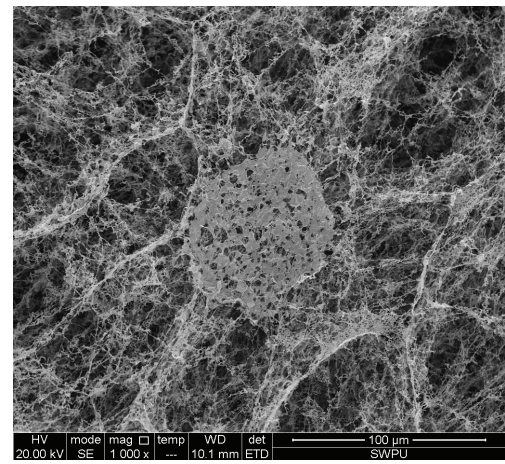

(e)

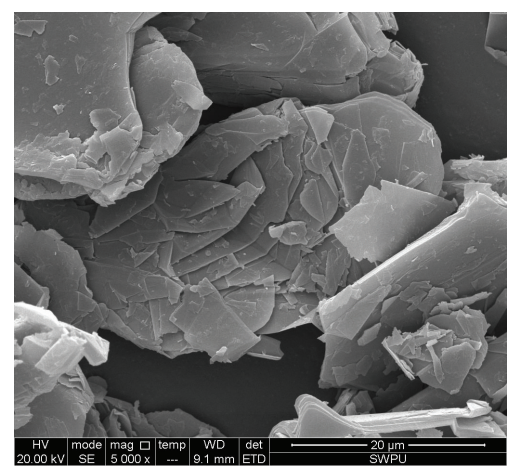

(c)

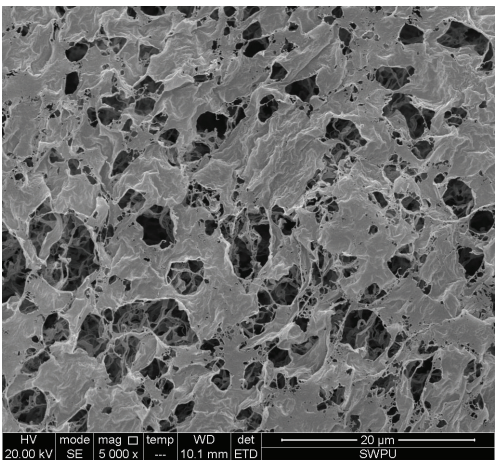

(f)

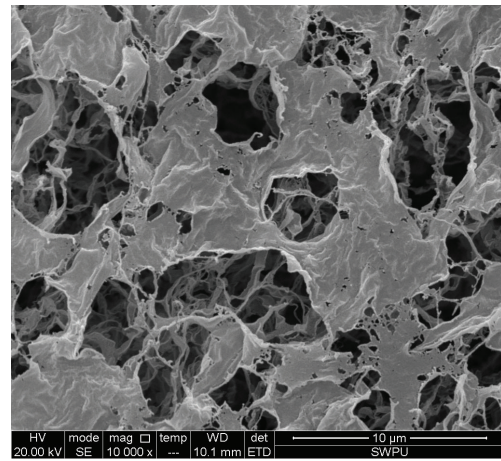

(g)

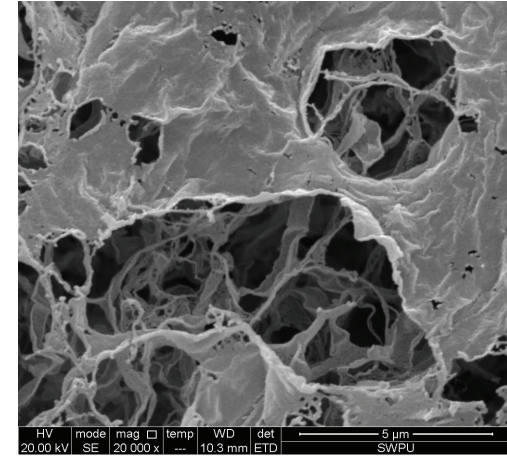

(h)

FIGURE 10: SEM images with different magnifications of graphite $(\mathrm{a}-\mathrm{d})$ and GO dispersion $(\mathrm{e}-\mathrm{h})$ interior microstructures.

which attributable to carbon and oxygen, respectively. The C1s XPS spectra of GO were depicted in Figure 9(b). It was clear that four different types of carbon with chemical environments were obvious in $\mathrm{GO}$ : carbon $\mathrm{sp}^{2}(\mathrm{C}=\mathrm{C}, 284.6 \mathrm{eV})$, epoxy/hydroxyl (C-O, 286.5 eV), carbonyl $(\mathrm{C}=\mathrm{O}, 287.8 \mathrm{eV})$, and carboxyl $(\mathrm{O}-\mathrm{C}=\mathrm{O}, 288.7 \mathrm{eV})$, respectively. This could be attributed to the fact that a large amount of surface oxide groups were chemically attached to the defect or edge sites of the basal planes [26].

The microstructure of graphite was composed of numerous graphite sheets which were clearly showed in Figures $10(a)-10(d)$. The interior microstructure of as-prepared GO dispersion was imaged by SEM of its lyophiled samples. According to SEM images (Figures 10(e)-10(h)), the GO aqueous dispersion had an obvious $3 \mathrm{D}$ porous network structure. The pore diameters ranged from submicrometer to several micrometers and the pore walls consisted of very thin layers of stacked GO sheets [27]. Therefore, it was reasonable to conclude that a loose dynamic GO network existed in the original dispersion of GO sheets owing to a force balance between electrostatic repulsion and binding interactions (hydrogen bonding, $\pi$-stacking, hydrophobic effect, etc.) [28].

The core flooding experiments were carried out to evaluate the ability of profile control of GO dispersion. Resistance factor and residual resistance factor were the important indicators that characterize abilities of liquid fluidity control and the permeability descent. Resistance factor was a measure of the relative mobility of profile control system, the greater resistance factor was the stronger the profile control system 


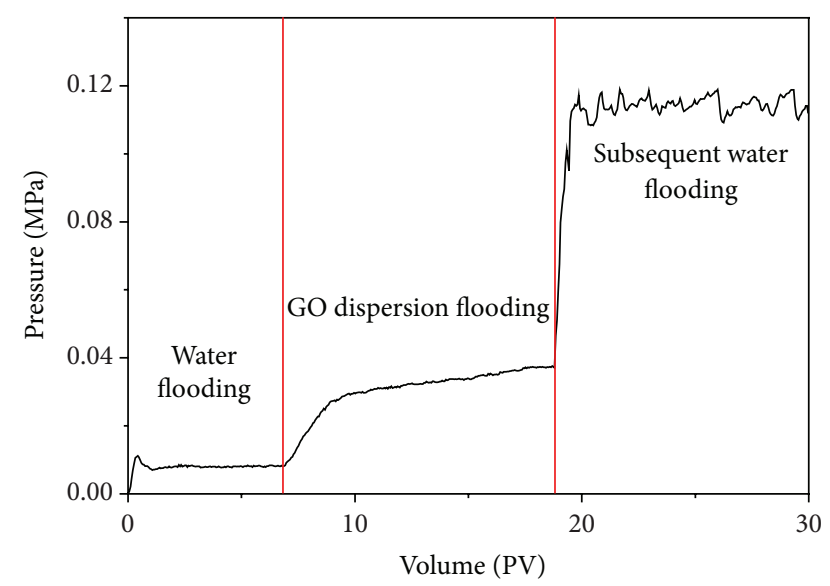

Figure 11: Pressure curve of flooding of GO dispersion with single core.

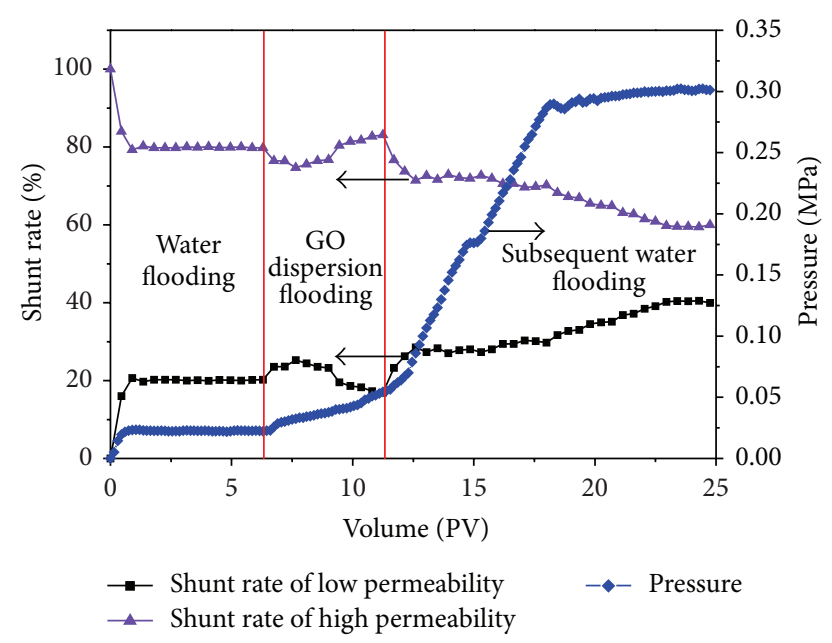

FIGURE 12: Shunt rate and pressure of flooding of GO dispersion with two cores in parallel.

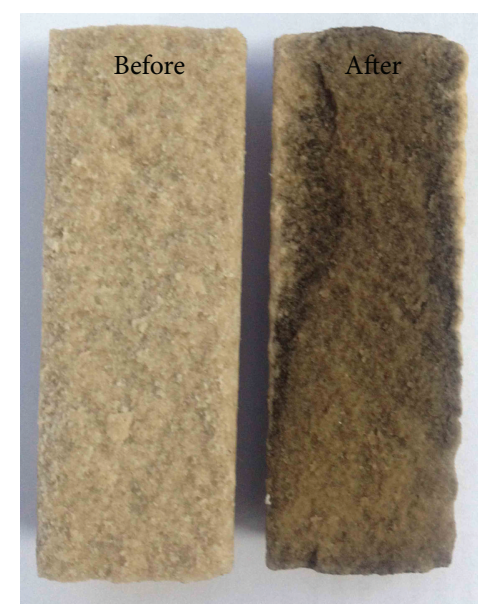

Figure 13: Profile image of the core before and after flooding. reduced the flow ability of fluid in porous media. And residual resistance factor was a measurement of the permeability reduction of profile control system permanently. The GO dispersion would produce two important effects after being injected into core: one is that it would increase the aqueous phase injection pressure; the other one is that GO's adsorption retention would cause the reduction of reservoir permeability. Those two effects led to the significant reduction of displacing phase fluidity in the oil reservoir. Therefore, the GO dispersion injection would reduce total fluidity of water in flooding layer with high permeability, lessen the gap of waterline advancement speed between the layers with higher and lower permeability, adjust water injection profile, and improve conformance factor. The pressure curve of single core flooding was showed in Figure 11. According to the results of injection pressure, resistance factor, and residual resistance factor of GO dispersion were calculated with

$$
\begin{gathered}
F_{\mathrm{R}}=\frac{\Delta P_{p}}{\Delta P_{\mathrm{wi}}}, \\
F_{\mathrm{RR}}=\frac{\Delta P_{\mathrm{wa}}}{\Delta P_{\mathrm{wi}}},
\end{gathered}
$$

where $F_{\mathrm{R}}$ was resistance factor, $F_{\mathrm{RR}}$ was residual resistance factor, $\Delta P_{\text {wi }}$ was stable pressure of precious water flooding (MPa), $\Delta P_{p}$ was stable pressure of $\mathrm{GO}$ dispersion flooding (MPa), $\Delta P_{\text {wa }}$ was stable pressure of subsequent water flooding $(\mathrm{MPa})$. The resistance factor of GO dispersion was 4.6 and the residual resistance factor was 14.3 , respectively, which indicated that GO dispersion had an ability to adjust the profile of fluid absorption.

The result of flooding experiment of two cores in parallel was showed in Figure 12 and the shunt rate of low permeability was $20 \%$ approximately in water flooding stage, which was in accord with the permeability ratio of the cores $(1: 4)$. In GO dispersion flooding stage, the GO dispersion, which had an obvious 3D porous network structure (according to SEM images), flowed into high permeability layer preferentially. Graphene oxide nanosheets could absorb on surface of the core (Figure 13) and cause a certain extent physical plugging effect. GO dispersion plugged the high permeability layer and caused an increase of injection pressure, but the high permeability layer was not been plugged completely, the penetrability of high permeability layer was reduced but the reservoir had a certain extent connectivity because of the porous structure of GO dispersion. The shunt rate of low permeability increased from $20 \%$ to $40 \%$ around in the subsequent water flooding. So we demonstrated that the GO dispersion has an ability to adjust water injection profile, reduce permeability ratio, and improve conformance factor.

\section{Conclusions}

In conclusion, a simple chemical route has been developed for the preparation of water dispersed nanosized graphene oxide, which cancelled high-temperature stage and prolonged the reaction time of mid-temperature to improve the extent of oxidation of graphite and avoid the damage of eruption in high-temperature reaction. The method developed 
here is simple, scalable, and environmental friendly. FTIR, XRD, Ultraviolet-visible, TGA, Raman spectrum, and XPS measurements indicate that the GO has been synthesized successfully. GO is essentially a single atomic sheet, while its lateral dimension extends to the size of colloidal particles. SEM images have showed the microcosmic structure of the GO dispersion that GO sheets with lateral dimensions of several micrometers are able to contact with each other to form a loose network in solution. Flooding experiments show that the GO dispersion has an ability to adjust water injection profile, reduce permeability ratio, and improve conformance factor, and could be used as profile control agent in the oilfield water-flooding. Our work may lay a foundation for further industrial applications of GO in oilfield exploitation.

\section{Conflict of Interests}

The authors declare that there is no conflict of interests regarding the publication of this paper.

\section{Acknowledgments}

This work was supported by the National Science and Technology Major Project of China (no. 2011ZX05024-004). The experiments were performed at the State Key Laboratory of Oil and Gas Reservoir Geology and Exploitation of Southwest Petroleum University in Chengdu in China.

\section{References}

[1] Y. Si and E. T. Samulski, "Synthesis of water soluble graphene," Nano Letters, vol. 8, no. 6, pp. 1679-1682, 2008.

[2] K. S. Novoselov, A. K. Geim, S. V. Morozov et al., "Electric field in atomically thin carbon films," Science, vol. 306, no. 5696, pp. 666-669, 2004.

[3] E. J. Yoo, J. Kim, E. Hosono, H.-S. Zhou, T. Kudo, and I. Honma, "Large reversible Li storage of graphene nanosheet families for use in rechargeable lithium ion batteries," Nano Letters, vol. 8, no. 8, pp. 2277-2282, 2008.

[4] S. Park and R. S. Ruoff, "Chemical methods for the production of graphenes," Nature Nanotechnology, vol. 4, no. 4, pp. 217-224, 2009.

[5] A. K. Geim, “Graphene: status and prospects," Science, vol. 324, no. 5934, pp. 1530-1534, 2009.

[6] L. J. Cote, J. Kim, V. C. Tung, J. Luo, F. Kim, and J. Huang, "Graphene oxide as surfactant sheets," Pure and Applied Chemistry, vol. 83, no. 1, pp. 95-110, 2011.

[7] K. S. Kim, Y. Zhao, H. Jang et al., "Large-scale pattern growth of graphene films for stretchable transparent electrodes," Nature, vol. 457, no. 7230, pp. 706-710, 2009.

[8] B. Hsia, N. Ferralis, D. G. Senesky, A. P. Pisano, C. Carraro, and R. Maboudian, "Epitaxial graphene growth on 3C-SiC(111)/AlN(0001)/Si(100)," Electrochemical and Solid-State Letters, vol. 14, no. 2, pp. K13-K15, 2011.

[9] Z. Sun, Z. Yan, J. Yao, E. Beitler, Y. Zhu, and J. M. Tour, "Growth of graphene from solid carbon sources," Nature, vol. 468, no. 7323, pp. 549-552, 2010.

[10] B. C. Brodie, "On the atomic weight of graphite," Philosophical Transactions of the Royal Society of London, vol. 149, pp. 249259, 1859.
[11] W. S. Hummers Jr. and R. E. Offeman, "Preparation of graphitic oxide," Journal of the American Chemical Society, vol. 80, no. 6, p. $1339,1958$.

[12] S. Stankovich, D. A. Dikin, R. D. Piner et al., "Synthesis of graphene-based nanosheets via chemical reduction of exfoliated graphite oxide," Carbon, vol. 45, no. 7, pp. 1558-1565, 2007.

[13] S. Stankovich, R. D. Piner, S. T. Nguyen, and R. S. Ruoff, "Synthesis and exfoliation of isocyanate-treated graphene oxide nanoplatelets," Carbon, vol. 44, no. 15, pp. 3342-3347, 2006.

[14] S. Stankovich, R. D. Piner, X. Chen, N. Wu, S. T. Nguyen, and R. S. Ruoff, "Stable aqueous dispersions of graphitic nanoplatelets via the reduction of exfoliated graphite oxide in the presence of poly(sodium 4-styrenesulfonate)," Journal of Materials Chemistry, vol. 16, no. 2, pp. 155-158, 2006.

[15] A. Lerf, H. He, M. Forster, and J. Klinowski, "Structure of graphite oxide revisited," The Journal of Physical Chemistry B, vol. 102, no. 23, pp. 4477-4482, 1998.

[16] W. W. Cai, R. D. Piner, F. J. Stadermann et al., "Synthesis and solid-state NMR structural characterization of 13C-labeled graphite oxide," Science, vol. 321, no. 5897, pp. 1815-1817, 2008.

[17] Z.-H. Liu, Z.-M. Wang, X. Yang, and K. Ooi, "Intercalation of organic ammonium ions into layered graphite oxide," Langmuir, vol. 18, no. 12, pp. 4926-4932, 2002.

[18] P. Liu, K. Gong, P. Xiao, and M. Xiao, "Preparation and characterization of poly(vinyl acetate)-intercalated graphite oxide nanocomposite," Journal of Materials Chemistry, vol. 10, no. 4, pp. 933-935, 2000.

[19] S. Park, K. Lee, G. Bozoklu, W. W. Cai, S. T. Nguyen, and R. S. Ruoff, "Graphene oxide papers modified by divalent ionsenhancing mechanical properties via chemical cross-linking," ACS Nano, vol. 2, no. 3, pp. 572-578, 2008.

[20] M. Hirata, T. Gotou, and M. Ohba, "Thin-film particles of graphite oxide. 2: preliminary studies for internal micro fabrication of single particle and carbonaceous electronic circuits," Carbon, vol. 43, no. 3, pp. 503-510, 2005.

[21] L. J. Cote, F. Kim, and J. Huang, "Langmuir-blodgett assembly of graphite oxide single layers," Journal of the American Chemical Society, vol. 131, no. 3, pp. 1043-1049, 2009.

[22] H. Remy and J. Kleinberg, Treatise on Inorganic Chemistry, Elsevier, Amsterdam, The Netherlands, 1956.

[23] W.-S. Ma, J. Li, B.-J. Deng, and X.-S. Zhao, "Preparation and characterization of long-chain alkyl silane-functionalized graphene film," Journal of Materials Science, vol. 48, no. 1, pp. 156-161, 2013.

[24] C. Zhu, S. Guo, Y. Fang, and S. Dong, "Reducing sugar: new functional molecules for the green synthesis of graphene nanosheets," ACS Nano, vol. 4, no. 4, pp. 2429-2437, 2010.

[25] K. N. Kudin, B. Ozbas, H. C. Schniepp, R. K. Prud'homme, I. A. Aksay, and R. Car, "Raman spectra of graphite oxide and functionalized graphene sheets," Nano Letters, vol. 8, no. 1, pp. 36-41, 2008.

[26] C.-T. Hsieh and W.-Y. Chen, "Water/oil repellency and work of adhesion of liquid droplets on graphene oxide and graphene surfaces," Surface and Coatings Technology, vol. 205, no. 19, pp. 4554-4561, 2011.

[27] Y. X. Xu, Q. Wu, Y. Q. Sun, H. Bai, and G. Q. Shi, “Threedimensional self-assembly of graphene oxide and DNA into multifunctional hydrogels," ACS Nano, vol. 4, no. 12, pp. 73587362,2010

[28] H. Bai, C. Li, X. Wang, and G. Shi, "On the gelation of graphene oxide," The Journal of Physical Chemistry C, vol. 115, no. 13, pp. 5545-5551, 2011. 

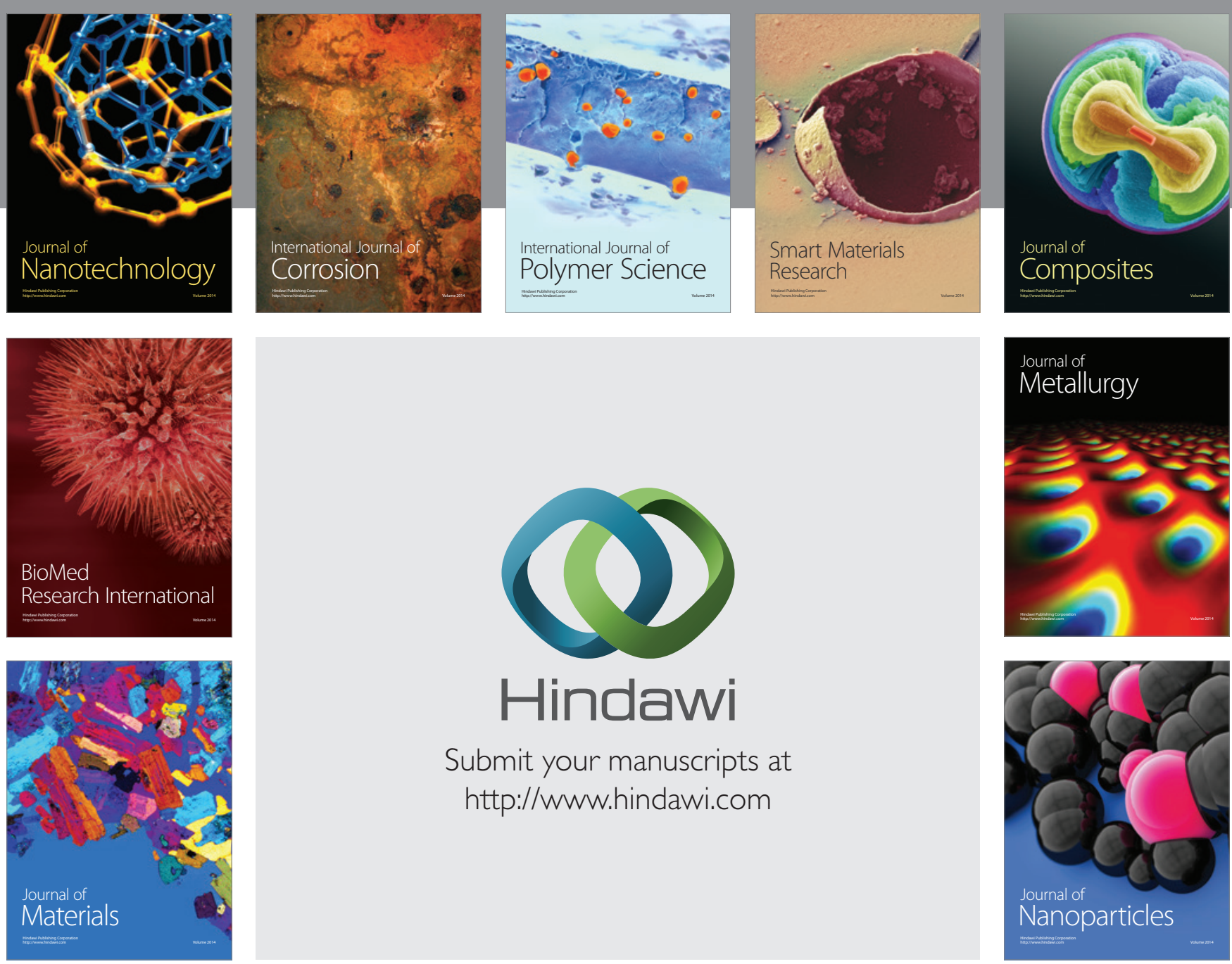

Submit your manuscripts at http://www.hindawi.com
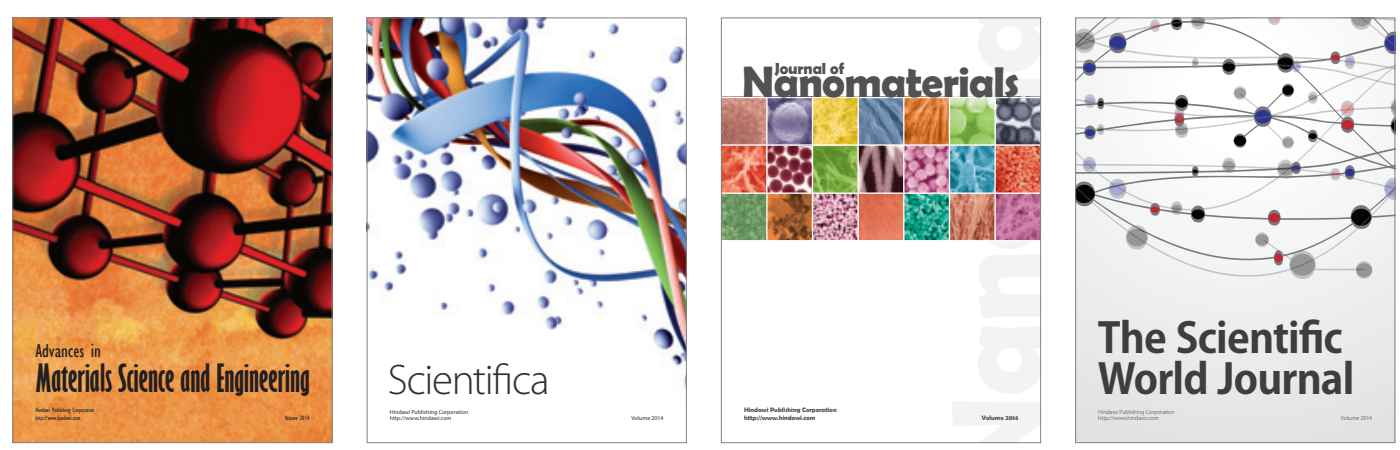

\section{The Scientific World Journal}
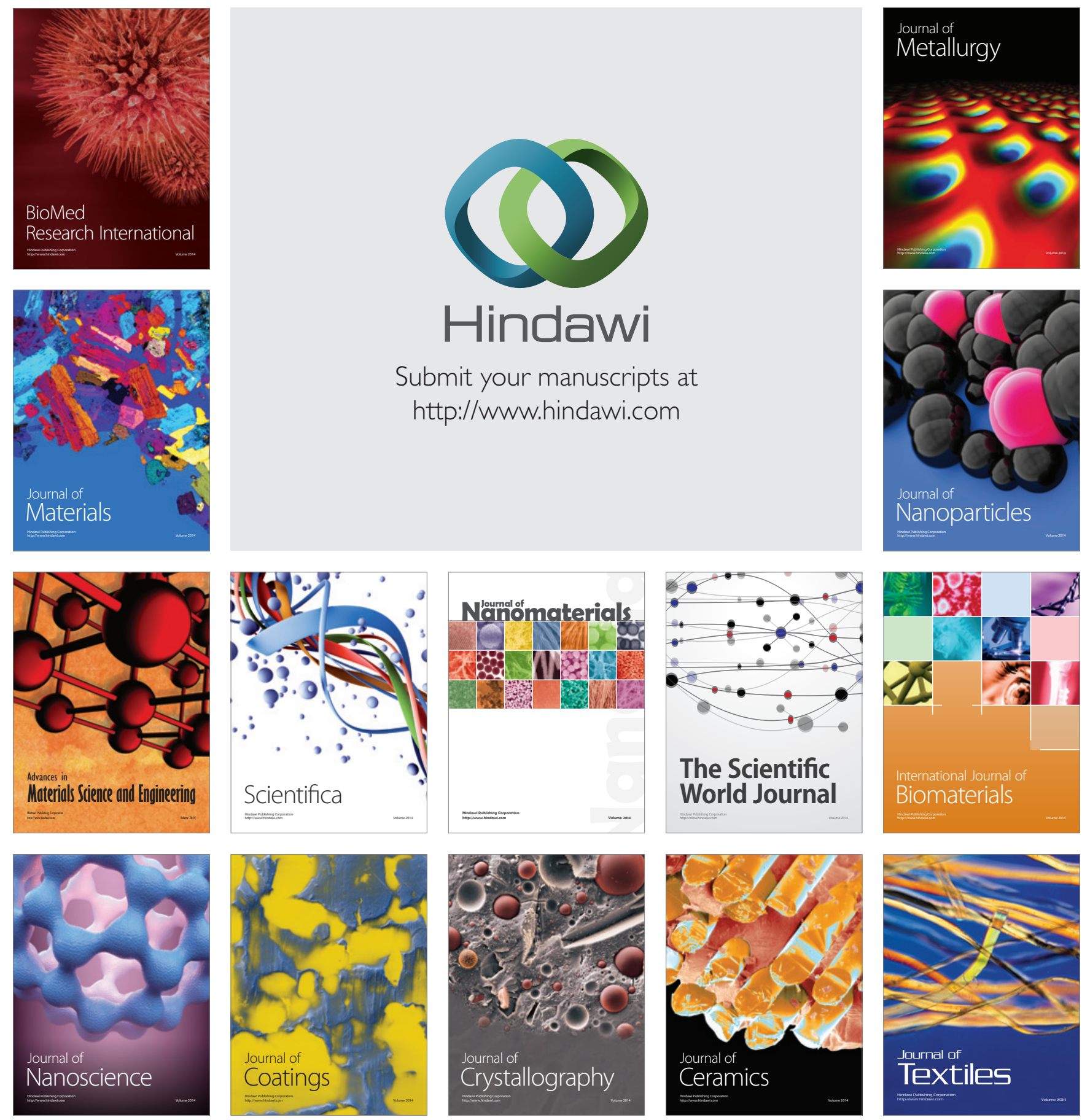